\title{
Cloning and characterization of two subunits of calcineurin cDNA in naked carp (Gymnocypris przewalskii) from Lake Qinghai, China
}

\author{
Fulei Wei ${ }^{1}$, Chenlong Wang ${ }^{1}$, Zhaoxi Wang ${ }^{1}$, Yawei Kong ${ }^{2}$, Yuan Shi ${ }^{1}$, Jianquan Shi ${ }^{3}$, \\ Delin $\mathrm{Qi}^{4}$, Hongfang $\mathrm{Qi}^{3}$, Jian Liang ${ }^{1}$, Rongqing Zhang ${ }^{1}$, Changzhong Li ${ }^{1}$
}

\author{
${ }^{1}$ College of Eco-Environmental Engineering, Qinghai University, Xining, China \\ ${ }^{2}$ Centre for Regenerative Medicine, Massachusetts General Hospital, Harvard Medical School, \\ Boston, USA \\ ${ }^{3}$ Emergency Centre of Gymnocypris przewalskii, Xining, China \\ ${ }^{4}$ College of Agriculture and Animal Husbandry, Qinghai University, Xining, China
}

\begin{abstract}
The naked carp (Gymnocypris przewalskii), a native teleost, plays an important role in maintenance of the ecological balance in the system of Lake Qinghai (altitude, $3.2 \mathrm{~km}$ ) on the Qinghai-Tibet Plateau in China. Calcineurin $(\mathrm{CN})$ is the only member of the serine/threonine phosphatase family that can be activated by both $\mathrm{Ca}^{2+}$ and calmodulin (CaM) and involved in many important physiological processes such as salt tolerance/ /adaption. In this report, cDNAs of $\mathrm{CN}$ catalytic subunit paralogue isoforms: $G p C A \alpha$ (GenBank accession no. JQ407043), $G p C A \gamma$ (GenBank accession no. JQ407043), and CN regulatory subunit ( $G p C B$ ) (GenBank accession no. JQ410473), were isolated from Gymnocypris przewalskii and their expression patterns in embryos development were characterized. Gene expression profile demonstrated that $G p C A$ and $G p C B$ mRNA was distributed ubiquitously in all embryonic stages and showed decline until final stage of development. Immunohistological analysis revealed CN localization in different tissues including kidney, heart, brain, spermary, and gill. Collectively, these results provide molecular basis and clues to further understand the role of $\mathrm{CN}$ during embryos development and its function in tissues for the adaptation mechanism of naked carp. (Folia Histochemica et Cytobiologica 2014, Vol. 52, No. 3, 232-243)
\end{abstract}

Key words: Gymnocypris przewalskii; calcineurin; embryogenesis; calcineurin subunits; cloning; cDNA; IHC; Qinghai Lake

\section{Introduction}

Lake Qinghai is a saltwater lake $[\mathrm{pH} 9.4$, titraTab. alkalinity $\left(30 \mathrm{mmol} / \mathrm{l}^{-1}\right), \mathrm{Mg}^{2+}$-rich $\left(18.7 \mathrm{mmol} / \mathrm{l}^{-1}\right)$, $\mathrm{Ca}^{2+}$-poor $\left(0.30 \mathrm{mmol} / \mathrm{l}^{-1}\right)$ and saline $(9$ per thousand $\left.)\right]$ located within the Qiadam Basin of the Qinghai-Tibetan Plateau of western China at an elevation of $3200 \mathrm{~m}$ [1]. The naked carp, Gymnocypris przewalskii, mainly distributed in the Lake Qinghai, has always

Correspondence address: $\mathrm{Ch}$. Li, Ph.D.

College of Eco-Environmental Engineering

Qinghai University, Xining 810016, China

tel./fax: +86 9715310586

e-mail: lichangzhong@qhu.edu.cn received much attention, not only because of its fishery importance but also its ecological significance in the fish-bird-grassland system around Lake Qinghai. Every year the migrant birds from other parts of the world breed in the spring in the Bird Island in Lake Qinghai and around its adjoining freshwater rivers. Approximately 3000 tons of naked carp were captured by birds as their feed. The commercial fishing of Gymnocypris przewalskii from the 1950s until the 1990s was forced to be stopped for the sharp decrease of fish stocks. In the past fifty years, the total amount reserves has decreased from $20 \times 10^{4}$ tons in 1960 to less than $0.5 \times 10^{4}$ tons now, especially because of decreasing water level and increasing salinity [2-4]. The naked carp has to face critical challenges such 
as the increasing salinity levels of the lake, which is mainly caused by continuously decreased water levels at a rate of 10-12 cm per year over the last 50 years [5]. The increase of salinity began since 1978 and its rate is currently accelerating [6, 7]. Further, another challenge for the naked carp is the salinity changes during their annual migration from the saline lake to adjoining freshwater rivers including Quanjihe river, Shaliuhe river, Heimahe river and Buhahe river, where they spawn and then return to the lake [4]. At present, the basic biological adaptation mechanism of naked carp to the living condition, including low temperature, saline water, and hypoxia, has not been fully investigated. Therefore, understanding the embryonic developmental process of naked fish in freshwater and the adaptation mechanisms to saline lake water will be of great help for the artificial reproduction of naked carp, wild stocks recovery, and the ecosystem protection around Lake Qinghai.

Calcineurin $(\mathrm{CN})$, which is composed of two subunits: catalytic subunit (Calcineurin A, CNA) and regulatory subunit (Calcineurin $\mathrm{B}, \mathrm{CNB}$ ), is the only member of the serine/threonine phosphatase family that can be activated by both $\mathrm{Ca}^{2+}$ and calmodulin (CaM) [8-10]. Calcineurin has been proven to be a multifunctional enzyme and plays critical roles in regulating various cellular activities through direct dephosphorylation of the target proteins. After activation by both $\mathrm{Ca}^{2+}$ and $\mathrm{CaM}, \mathrm{CN}$ dephosphorylates its cytoplasmic substrate, the nuclear factor of activated $\mathrm{T}$ cells (NFAT). The activated NFATc translocates from the cytosol into the nucleus to control the expression of target genes. Previous studies showed that, in the sea urchin egg, a high intracellular calcium increase after egg fertilization has been observed [11]. It was also found that $\mathrm{CN}$ mRNAs are expressed maximally just before full maturation of the testis [12], and CNA was highly expressed in the scallop gonad [13]. Recently, it has been demonstrated that CN-NFAT signalling regulates the early lineage specification in mouse embryos [14]. Balanced Ca/NFAT signalling in progenitor cells of the developing embryo is essential, while inappropriate activation of NFAT is deleterious in embryonic development [15]. Many data suggest that $\mathrm{CN}$ is involved in the embryonic development; however, the possible mechanism of $\mathrm{CN}$ in controlling differentiation remains largely unknown.

In this study, cDNAs sequences of $\mathrm{CN}$ catalytic subunit and $\mathrm{CN}$ regulatory subunit were isolated from Gymnocypris przewalskii, and their expression patterns at different embryonic developmental stages and localization in different tissues were analysed. These results provide some important clues to further un- derstand the diverse roles of $\mathrm{CN}$ in the development of naked carp.

\section{Material and methods}

Animals. Live individuals of artificially cultivated 2 years old naked carp (20-25 cm in length and 100-150 g in wet weight) were supplied by the Rescue Centre of Gymnocypris przewalskii (Qinghai, China) and cultured in an aquarium with flowing freshwater in the laboratory. In order to avoid stress reaction, the naked carps were kept 3-5 days for acclimatization before being used. These animals were used for cloning of subunits of $\mathrm{CN}$ cDNAs.

Preparation of zygotes. Germ cells were collected from wild adult in Quanjihe river (Qinghai, China) in June and July during spawning migration period. Zygotes were incubated in flowing water (the water temperature maintained $\left.10-15^{\circ} \mathrm{C}\right)$ from Shaliuhe river $\left(\mathrm{K}^{+}, 8 \mathrm{mg} / \mathrm{L} ; \mathrm{Na}^{+}\right.$, $18 \mathrm{mg} / \mathrm{L} ; \mathrm{Ca}^{2+}, 45 \mathrm{mg} / \mathrm{L} ; \mathrm{Mg}^{2+}, 15 \mathrm{mg} / \mathrm{L} ; \mathrm{Cl}^{-}, 15 \mathrm{mg} / \mathrm{L} ; \mathrm{SO}_{4}^{2-}$, $15 \mathrm{mg} / \mathrm{L} ; \mathrm{HCO}_{3}^{-}, 200 \mathrm{mg} / \mathrm{L}$ ) after artificial fertilization in order to simulate the wild hatching condition. The zygotes at different embryonic stages were collected and stored in liquid nitrogen.

RNA extraction and cDNA synthesis. Total RNA was extracted with Trizol (Invitrogen, Carlsbad, CA, USA) agent from gill and zygotes. For calcineurin subunits fragments cloning, single strand cDNA was reverse transcribed from total RNA extracted from gill using PrimeScript RTase Kit (Takara, Kyoto, Japan).

Isolation and sequencing of calcineurin subunits. Total RNA from gill of was reverse transcribed to single strand cDNA, this cDNA was used for CN cDNA fragment cloning as template. In order to avoid the sequence errors and individual difference, we repeated the reverse transcription more than three times and the gills were from different individuals. $G p \mathrm{CA} \alpha$ cDNA fragment was amplified using the following primers: GpCAF53 (forward) and GpCAR53 (reverse) (Table 1), which were designed according to the conserved region of Oncorhynchus mykis calcineurin catalytic subunits (CNA) cDNA. 5' untranslated region of GpCA $\alpha$ cDNA was isolated using Nested PCR method. The first PCR reaction was performed using gene specific primer GpCA $\alpha$ AP1 (Table 1) and 5' RACE (Rapid-Amplification of cDNA Ends) Outer Primer. The Nested PCR reaction was carried out using gene specific primer GpCA $\alpha$ AP2 (Table 1) and 5' RACE inner Primer following the introduction. For the 3' untranslated region of $G p \mathrm{CA} \alpha \mathrm{cDNA}$, the first $\mathrm{PCR}$ reaction was performed using gene specific primer GpCA $\alpha$ SP1 (Table 1) and 3' RACE Outer Primer, then the Nested PCR Reaction was performed using gene specific primer GpCA $\alpha \mathrm{SP} 2$ (Table 1) and 3' RACE inner primer 
Table 1. Primer names and primer sequences used for PCR

\begin{tabular}{|c|c|c|c|}
\hline Primer names & Sequences & Application & Efficiency \\
\hline GpCAF53 & ATCAACCATCCCACCAC & & \\
\hline GpCAR53 & TCTTCTCAGAGCCGTAGTC & & \\
\hline $\mathrm{GpCA} \alpha \mathrm{AP} 1$ & GACCACCGTGTACGCAGAGGAACT & 5'RACE & \\
\hline $\mathrm{GpCA} \alpha \mathrm{AP} 2$ & GGAAGAGGGTGGTGGGATGGTTGA & 5'RACE & \\
\hline $\mathrm{GpCA} \alpha \mathrm{SP} 1$ & ССАТСССАССАСССТСТТССТССТС & 3'RACE & \\
\hline $\mathrm{GpCA} \alpha \mathrm{SP} 2$ & CCTCTGCGTACACGGTGGTCTC & 3'RACE & \\
\hline $\mathrm{GpCA} \alpha \mathrm{CF} 1$ & TGGCTAAGAAACCCTGACTGAACTG & Confirm PCR & \\
\hline $\mathrm{GpCA} \alpha \mathrm{CR} 1$ & TGCAGCATCTTAAACCCACATCTC & Confirm PCR & \\
\hline GpCAF55 & GTGTATGATGCGTGTATGG & & \\
\hline GpCAR55 & GTTGGGCAGCCAGTAG & & \\
\hline GpCA $\gamma \mathrm{AP} 1$ & TCCTGGGCTTCATGTGCTCTTAT & 5'RACE & \\
\hline GpCA $\gamma \mathrm{AP} 2$ & AACGCACAGGAACTGCTGGTTGAG & 5'RACE & \\
\hline GpCA $\gamma \mathrm{SP} 1$ & ATAAGAGCACATGAAGCCCAGGAT & 3'RACE & \\
\hline GpCA $\gamma \mathrm{SP} 2$ & AGTCAGACGACAGGCTTCCC & 3'RACE & \\
\hline $\mathrm{GpCA} \gamma \mathrm{CF} 1$ & TTTCCGTTTCTCCGCTCTAACA & Confirm PCR & \\
\hline GpCA $\gamma \mathrm{CR} 1$ & CACCACCCCAATGCTAATGTCTAT & Confirm PCR & \\
\hline GpCBF17 & CGAGGGAGTCTCACAGTTC & & \\
\hline GpCBR17 & CGTCCACCACCATCTTTT & & \\
\hline GpCBAP1 & CTGGGTGTCCTTCAGGTTATTCC & 5'RACE & \\
\hline GpCBAP2 & TGTCATAAATCCTGAACGCAAAGC & 5'RACE & \\
\hline GpCBSP1 & CGAGGGAGTCTCACAGTTC & 3'RACE & \\
\hline GpCBSP2 & GCTCCGCTTTGCGTTCAGGATTTATG & 3'RACE & \\
\hline GpCBCF1 & CAAGGCGAAGACCAGCCAGAAT & Confirm PCR & \\
\hline GpCBCR1 & TGAAAAGAGGGTGCTCGGTGAA & Confirm PCR & \\
\hline GpCA $\alpha$ sqpF3 & GCCCGCTGGTCTGGATT & Real time PCR & $91 \%$ \\
\hline GpCA $\alpha$ sqpR3 & CACAGTTCAGTCAGGGTTTCTT & Real time PCR & \\
\hline GpCA $\gamma \mathrm{sqpF} 3$ & GCGGGCTAACGCGACTA & Real time PCR & $99 \%$ \\
\hline GpCA $\gamma \mathrm{sqpR} 3$ & TGGGAGGGTACGGCACA & Real time PCR & \\
\hline GpCBRTF1 & GGGAATAACCTGAAGGACACC & Real time PCR & $92 \%$ \\
\hline GpCBRTR1 & GTCTAAGCCACCAACCACAA & Real time PCR & \\
\hline$\beta$-actinRTF1 & ATGGGAACCGCTGCCTCTT & Real time PCR & $99 \%$ \\
\hline$\beta$-actinRTR1 & GGTCAGCAATGCCAGGGTA & Real time PCR & \\
\hline GpCAexPF1 & CATGCCATGGGCACTAAAGAGCCGAAAG & Recombinant protein & \\
\hline GpCAexPR1 & CCGCTCGAGCTCGTTGGCAGCGTTAA & Recombinant protein & \\
\hline GpCBexPF1 & CATGCCATGGGAAATGAGGCGAGTTATCC & Recombinant protein & \\
\hline GpCBexPR1 & CCGCTCGAGCACGTCCACCACCATC & Recombinant protein & \\
\hline
\end{tabular}

following the instruction of 3' Full RACE Core Set Ver.2.0 (Takara, Kyoto, Japan). Finally, primers GpCA $\alpha \mathrm{CF} 1$ and $\mathrm{GpCA} \alpha \mathrm{CR} 1$ (Table 1) were used for confirmation of the $G p \mathrm{CA} \alpha$ coding sequence.

For $G p C A \gamma$ cDNA isolation, GpCAF55 (forward) and GpCAR55 (reverse) were used for fragment amplification
(Table 1). 5' untranslated region of GpCA $\gamma$ cDNA was isolated using gene specific primer GpCA $\gamma \mathrm{AP} 1$ (Table 1) and nested PCR primer GpCA $\gamma$ AP2 (Table 1). For the 3' untranslated regions of GpCA $\gamma$ cDNA, the PCR reactions were performed using gene specific primer GpCA $\gamma \mathrm{SP} 1$ (Table 1) and nested PCR primer GpCA $\gamma$ SP2 (Table 1). Con- 
firmation PCR was performed using primers $\mathrm{GpCA} \gamma \mathrm{CF} 1$ and GpCA $\gamma$ CR1 (Table 1).

For $G p \mathrm{CB}$ cDNA fragment, degenerate oligonucleotide primers GpCBF17 (forward) and GpCBR17 (reverse) (Table 1) were used to obtain an expected product. 5' untranslated region of $\mathrm{GpCB} \mathrm{cDNA}$ was isolated using two primers GpCBAP1 and GpCBAP2 (Table 1). Meanwhile, GpCBSP1 and nested primer GpCBSP2 (Table 1) were used to obtain 3' untranslated region. Finally, primers GpCBCF1 and GpCBCR1 (Table 1) were used to confirm the nucleotide sequence of $\mathrm{GpCB}$.

In all the PCR reactions, the annealing temperature of the primers was $60^{\circ} \mathrm{C}$, and the elongation time were set as 1 min per kilobase DNA.

Sequence analysis. All PCR products were sequenced in Sangon Biotech Company (Shanghai, China). Homology analysis of the nucleotide and protein sequence was conducted with the BLAST algorithm at the National Centre for Biotechnology Information (http://www.ncbi.nlm.nih. gov/BLAST/). Vector NTI (InforMax, Frederick, USA) was used to perform multiple alignments and structure the phylogenetic tree with the NJ method.

Expression analysis of Calcineurin subunits during embryonic development. Real time PCR was carried out to determine the expression levels of calcineurin subunits in zygotes at different embryonic stages (hours post-fertilization, hpf): multicellular stage (3 hpf); blastula stage (13 hpf); gastrula stage (41 hpf); neurula stage (68 hpf); organogenesis ( $96 \mathrm{hpf}$ ). Total RNA was extracted with Trizol agent (Invitrogen, Carlsbad, USA) from zygotes, and was reverse transcribed to single strand cDNA. The cDNA was used as the template of RT-PCR. Primers (Table 1) were as follows: GpCA $\alpha$ sqpF3 and GpCA $\alpha$ sqpR3 (Table 1) (for $G p \mathrm{CA} \alpha$ ); GpCA $\gamma \mathrm{sqpF} 3$ and GpCA $\gamma$ sqpR3 (Table 1) (for $G p \mathrm{CA} \gamma$ ); GpCBRTF1 and GpCBRTR1 (Table 1) (for $G p C B$ ). $\beta$-actin, used as the positive control [16], was amplified with the primer pairs of $\beta$-actinRTF1 and $\beta$-actinRTR1 (Table 1 ). For real time PCR, it is carried out on IQ5 Real-time Quantitative PCR instrument (Bio-Rad, Hercules, CA, USA). The real time PCR performed using $\mathrm{iQ}^{\mathrm{TM}} \mathrm{SYBR}^{\circledR}$ Green Supermix (Bio-Rad, Hercules, CA, USA) and the level of expression was analysed with Livak's method. The thermocycler was programmed for $94^{\circ} \mathrm{C}$ denaturation for 2 min followed by 40 cycles of $94^{\circ} \mathrm{C}$ denaturation for $20 \mathrm{~s}, 56^{\circ} \mathrm{C}$ annealing for $20 \mathrm{~s}$, and $72^{\circ} \mathrm{C}$ for $20 \mathrm{~s}$. The reaction system including: $\mathrm{iQ}^{\mathrm{TM}}$ $\mathrm{SYBR}^{\circledR}$ Green Supermix $10 \mu \mathrm{l}$, cDNA $(1 \mu \mathrm{g} / \mu \mathrm{l}) 1 \mu \mathrm{l}$, reverse primer $(10 \mu \mathrm{M}) 0.5 \mu \mathrm{l}$, forward primer $(10 \mu \mathrm{M}) 0.5 \mu \mathrm{l}$ and $\mathrm{ddH}_{2} \mathrm{O} 8 \mu \mathrm{l}$.

Expression of GpCA in $E$. coli and polyclonal antibody preparation. Fragment of $\mathrm{GpCA} \alpha \mathrm{cDNA}$ was amplified with gene-specific primers GpCAexPF1 (forward) containing an
NcoI site (underlined) and GpCAexPR1 (reverse) containing an XhoI site (underlined). The amplicon was inserted into expression vector pET-28b (Novagen, Darmstadt, Germany) to construct recombinant plasmid pET-28b/ /GpCA. The recombinant plasmids were amplified in E. coli strain DH5 $\alpha$, purified and sequenced to verify the validity of ORF before transformed into $E$. coli strain BL21 (DE3, Novagen). Protein expression was induced with $1 \mathrm{mM}$ isopropylthiogalactopyranoside (IPTG) at 37 degrees centigrade, until the optical density of the culture at $600 \mathrm{~nm}$ reached 0.6. After $3 \mathrm{hrs}$ of induction, bacterial cells were harvested by centrifuging at $8000 \mathrm{~g}$ for $5 \mathrm{~min}$. Most of the recombinant protein was expressed in form of inclusion body, which was subject to sonication on ice. The inclusion body was purified, dissolved in lysis buffer (containing $8 \mathrm{M}$ urea, $0.02 \mathrm{M} \mathrm{NaH}_{2} \mathrm{PO}_{4}, \mathrm{pH} 7.2,0.5 \mathrm{M}$ $\mathrm{NaCl}$ ) and filtrated with $0.45 \mu \mathrm{m}$ filter before applied to a Hi-Trap HP Ni affinity column (Bio-Rad). After elution, recombinant protein was analysed on $15 \%$ sodium dodecyl sulphate-polyacrylamide gel electrophoresis (SDS-PAGE), and then dialyzed against phosphate-buffered saline (PBS). Protein yields were measured by BCA assay kit (Pierce, Rockford, IL, USA). Purified polyclonal antibody against the purified recombinant proteins was obtained following the reported method [17-19]. The purified antibody was used for Western blotting analysis and immunohistochemistry.

\section{Immunohistochemical analysis of GpCA in different} tissues. Tissues were fixed in $4 \%$ paraformaldehyde, dehydrated in ethanol, cleared in xylene, and then embedded in paraffin blocks, $5-\mu \mathrm{m}$ sections were cut and mounted on slides coated with polylysine. Immunohistochemical staining was performed as follows: (a) paraffin sections were deparaffinised and rehydrated to water; (b) incubated in $3 \% \mathrm{H}_{2} \mathrm{O}_{2}$ for $10 \mathrm{~min}$, then washed with PBS 3 times for $15 \mathrm{~min}$; (c) slides were boiled in citric acid buffer solution for $20 \mathrm{~min}$, then washed with PBS for 3 times after cooled; (d) blocked with goat serum (10\% in PBS) for $15 \mathrm{~min}$; (e) Rabbit anti-GpCA polyclonal antibody (1:250 in PBS) was incubated at $4^{\circ} \mathrm{C}$ for 12 hours, then slides were rinsed with PBS; (f) Histostain ${ }^{\mathrm{TM}}$-Plus Kits (Invitrogen) and DAB kit (Boster, Wuhan, China) were used to detect the immune complex following instructions; (g) counterstained with haematoxylin.

Statistics. Data were expressed as means \pm SE (Standard Error of Mean) of three independent experiments; statistical analyses were performed with Minitab 16 program (Minitab, University Park, PA, USA). Comparisons between groups were analysed via ANOVA followed by Duncan's multiple range tests and $p<0.05$ was considered as statistically significant. 


\section{Results and analysis}

\section{Cloning and sequence analyses of $\mathrm{GpCA} \alpha$ and $G p C A \gamma$}

A 298 bp cDNA fragment of $G p C A \alpha$ was obtained after RT-PCR amplification using primers (GpCAF53 and GpCAR53). After rapid amplification of cDNA ends (RACE) with gene specific primers (GpCA $\alpha \mathrm{AP} 1$ and GpCA $\alpha \mathrm{AP} 2$ for 5'UTR, GpCA $\alpha \mathrm{SP} 1$ and $\mathrm{GpCA} \alpha \mathrm{SP} 2$ for 3'UTR) and confirmation PCR with gene specific primers ( $\mathrm{GpCA} \alpha \mathrm{CF} 1$ and $\mathrm{GpCA} \alpha \mathrm{CR} 1)$, the complete cDNA sequence of $G p C A \alpha$ was cloned (Figure 1A). The complete cDNA sequence contains a 726 bp 5' untranslated sequence, an ORF consisting of $1503 \mathrm{bp}$, a TAA stop codon, a 456 bp 3' untranslated sequence, and a poly (A) tail of 11 nucleotides. The deduced $G p C A \alpha$ protein consists of 500 amino acids with a molecular weight of $56,316 \mathrm{Da}$ and theoretical isoelectric point (pI) 5.60 according to ProtParam analysis [20].

In the process of $G p C A \gamma$ cDNA isolation, GpCAF55 and GpCAR55 were synthesized to amplify a 495 bp fragment. After 5'RACE with GpCA $\gamma$ AP1 and GpCA $\gamma$ AP2, 3'RACE with GpCA $\gamma$ SP1 and GpCA $\gamma \mathrm{SP} 2$, and a confirmation PCR with GpCA $\gamma \mathrm{CF} 1$ and GpCA $\gamma$ CR1, the complete 2685 bp cDNA sequence of $G p C A \gamma$ was isolated (Figure 1B). Structural analysis showed that it contains a $208 \mathrm{bp} 5$ ' untranslated sequence, an ORF of $1536 \mathrm{bp}$, a TAA stop codon, a 378 bp 3' untranslated sequence, and a poly (A) tail of 11 nucleotides. The deduced GpCA $\gamma$ protein consists of 511 amino acids with a molecular weight of 57,801.8 Da and theoretical pI 5.61.

After Conserved Domain Database search and ScanProsite annotation [21, 22], both GPCA $\alpha$ and $G p C A \gamma$ show a conserved metallophosphatase domain of Calcineurin and a serine/threonine specific protein phosphates signature (Figure 1).

In mammals, CNA is classified into three isoforms: $\alpha$-, $\beta$-, and $\gamma$-type. CNA $\alpha$ isoform is widely distributed; $\mathrm{CNA} \beta$ isoform has a conserved feature of an $\mathrm{N}$-terminal polyproline motif, whereas $\mathrm{CNA} \gamma$ is specifically expressed in the human testis [23]. Studies of the mRNAs encoding the different isoforms of the catalytic subunit (A subunit) of calcineurin revealed that they are expressed at different levels in rat thymus and kidney using in situ hybridization histochemistry with specific antisense oligonucleotide probes [24]. Immunocytochemical analysis revealed that both $\mathrm{CNA} \alpha$ and $\mathrm{CNA} \beta$ immunoreactivities differ in regional and cellular localizations in rat brain [25]. In addition, knockout mice lacking each of the isoforms had shown different dysfunctions, and the absence of $\mathrm{CNA} \alpha$ resulted in impaired kidney function [26, 27].
All the results indicated that each isoform plays its own function.

In gene bank, only two paralogue isoforms, $\alpha$ - and $\gamma$-type, have been characterized in Danio rerio and Salmo sala respectively, the accession numbers of Danio rerio are CAM46977.1 and NP_001074063.2, ACI67288.1 and ACI33493.1 for Salmo sala. The alignment between naked carp Gymnocypris przewalskii and Danio reri shows that the homology of $\mathrm{CNA} \alpha$ and CNA $\gamma$ is $93 \%$ and $94 \%$, respectively (Figure 2).

Notably, according to the phylogenetic tree analysis, the two CN A isoforms with the highest similarity in human is $\mathrm{HsCA} \gamma$. It is speculated that the variation of $G p C N A \alpha$ and $G p C N A \gamma$ formed after $G$. przewalskii species formation, and their functions need to be further investigated.

In naked carp, the two paralogue isoforms of CNA can be divided into 4 domains: catalytic core, CNB-binding domain, calmodulin-binding domain and inhibitory peptide. The paralogue alignment shows that the identity of the two protein sequences is $83 \%$. The identity is $90 \%$ for catalytic core domain, $96 \%$ for the CNB-binding domain, $100 \%$ for the calmodulin-binding domain and $86 \%$ for the inhibitory peptide (Figure 3). In addition, the poly-proline sequence, which may play a role in substrate recognition, was not found in the isoforms as that in the mammalian CNA $\beta$ isoform.

\section{Cloning and sequence analyses of $\mathrm{GpCB}$}

To clone the complete $G p C B$ cDNA sequence, GPCBF17 and GPCBR17 were utilized to gain a 243 bp fragment. Gene specific primers (GpCBAP1 and GpCBAP2) were used for 5'RACE, while gene specific primers (GpCBSP1 and GpCBSP2) for 3'RACE. In the end, a confirmation PCR was performed with gene specific primers (GpCBCF1 and GpCBCR1). The 1137 bp complete cDNA sequence of $G p C B$ contains 513 bp coding sequence (CDS) flanked by $47 \mathrm{bp}$ of 5' untranslated sequence and $577 \mathrm{bp}$ of 3' untranslated sequence; a TAA stop codon, and a poly (A) tail of 11 nucleotides (Figure 4).

As showed in Figure 4, the deduced 170 amino acids $\mathrm{GpCB}$ protein contains four conserved EF-hand type calcium-binding motifs including EF-1 (aa 31-42), EF-2 (aa 63-74), EF-3 (aa 100-111), and EF-4 (aa 141-152) with a calculated molecular mass of 19.3 $\mathrm{kDa}$ and an isoelectric point of 4.64. EF-hand proteins display a multitude of unique conformational states, together constituting a conformational continuum [28]. Calcium binding could induce the conformational change in the EF-hand motif, then leading to the activation of calcineurin. 
A

-726 G.thA

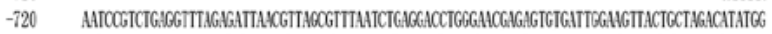

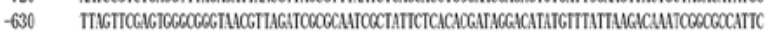

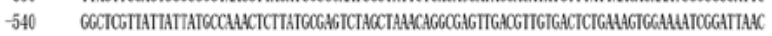

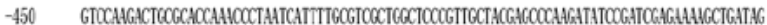

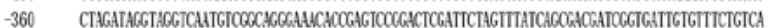
-270 GOAGGGGCALCGATOGAGTOOGTGTGTCTOCTAOCGTTGTTATTATGCTGCOCTGTGCOCATGTACACAACTGTTATTCAGTGTTGAC

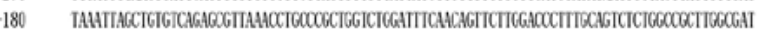

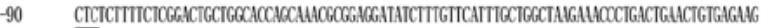

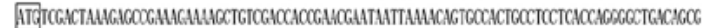
I S I K E P K E K L S I I E R I I K I I P L P P H Q G L T A

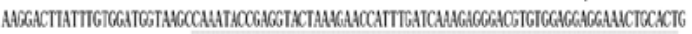
K D L F Y D G K P N T E I L K I H L I K E G R I E E E T A L

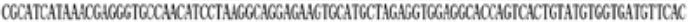
R I I I E G I I I L R O E K C I L E I E \& P I I I C G D I H

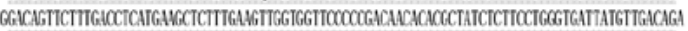
GQFFDLIKLFE IGGSPDNTRYLFLGDYYDR

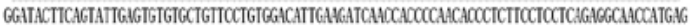
G Y F S I E C V L F L I I LK I I H P I T L F L LR G N HE

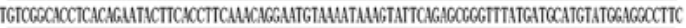
C R H L T E I F T F K Q E CKI K Y SER I I D A C N E A F GACTCCTTOOCTCTTOCAQCTCTTCTAHOCAGCAGTTCCTCTOGGTACAOCGOGGTCTCTCAOCAGHATCACTOCTTAGATGACATT D C L P L A A L L N Q Q F L C V H G G L S P E I N C L D D I

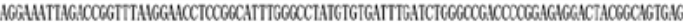
R K L D R F K E P P A F G P II C D L I I A D P P G E D Y G S E

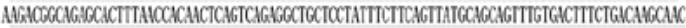
K T A E H F N H N S V R G C S Y F F S I A A Y C D F L T S I

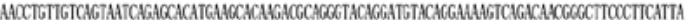
NLLS I I R A H E A Q D A G Y R VI R K S Q T T G F P S I

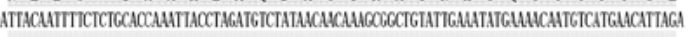
I T I F S A P N Y L D F Y N I K A A I L K Y E N N V N N I R

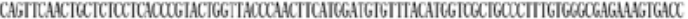
OF I C S P H P Y I P NF ID V F T IS L P F V G E K V T

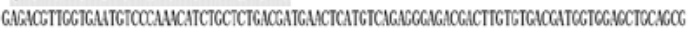
E T L I \& Y P N I C S D D E L N S E G D D L C D D G G A A A

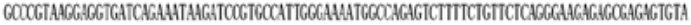
$A R K E Y I R$ \& I I R A I G K I A R I F S V L REE S E S I

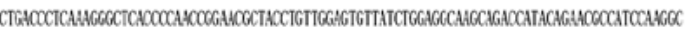
L T L K G L I P T G T L P V G V L S G G K Q I I Q N A I Q G

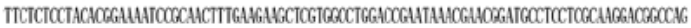

F S P T R E I R N F E E A R G L D R I \& E R I P P R K D G

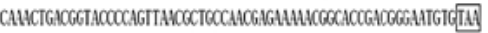

Q T D G T P I N A A N E K N G T D G N I *

GSGGAGGTTTGTACTTTIOCTCTOCTOCATAGCATOGTCTCTTTCTCTGCCACGTTCTACACAGHOOGHCOCTCACTCOOGGATCC

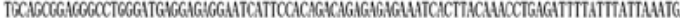

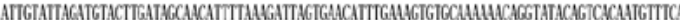

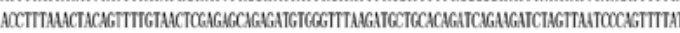

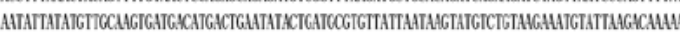
AHAA
B

$-208$

$-180$

$-90$

9.

6

27

271

36

121
451

541

181

631

$511 \quad \mathrm{~A} *$

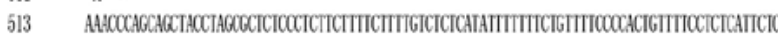

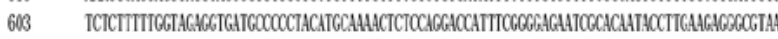

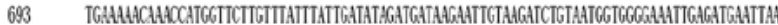

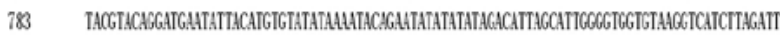
873 CAGTAGTALAHALA

Figure 1. cDNA sequences and deduced amino acid sequences of $G$. przewalskii. Calcineurin A alpha isoform and gamma isoform: $G p C A \alpha(\mathbf{A})$ and $G p C A \gamma(\mathbf{B}) . G p C A \alpha$ and $G p C A \gamma$ have putative metallophosphoesterase domains (shaded) including serine/threonine specific protein phosphatases signatures (in bold and italic)

Based on the reported data, CNB subunit shares high degree of conservation throughout evolution from yeast to mammalian, which makes it possible to functional interchange of CNB subunits between mammalian and $N$. crassa [29]. In this report, the sequence of $\mathrm{GpCB}$ shares various degree of similarity with those of CNB from other species (47\% identity to zebrafish, $48 \%$ to salmon, $92 \%$ to mollusk and $98 \%$ to rainbow trout).

\section{Gene expression profiles of calcineurin subunits during embryonic development}

Changes of intracellular free calcium concentration serve as a key signal (the second messenger) to modulate various physiological activities. In most eggs, ferti- lization triggers a propagating calcium explosion [30], which is demonstrated as a pre-requisite for egg activation [11]. In zebrafish, the defect in egg activation is a consequence of failure in $\mathrm{Ca}^{2+}$ wave induction [31]. In Xenopus embryos, calcineurin is transiently activated immediately after $\mathrm{Ca}^{2+}$ addition to a concentration that induces exit from metaphase arrest in meiosis II. Both the activation and subsequent inactivation of $\mathrm{CN}$ in fertilized eggs are crucial for the commencement of vertebrate embryonic development [32]. Injecting $\mathrm{CN}$ inhibitors into dorsal side can block dorsal-side signalling that affect late-stage development of the heart, kidney, liver, gut and somatic tissue during embryogenesis [33].

In our report, real time RT-PCR assay (Figure 5) demonstrated that the expression patterns of $G p C A \alpha$, 

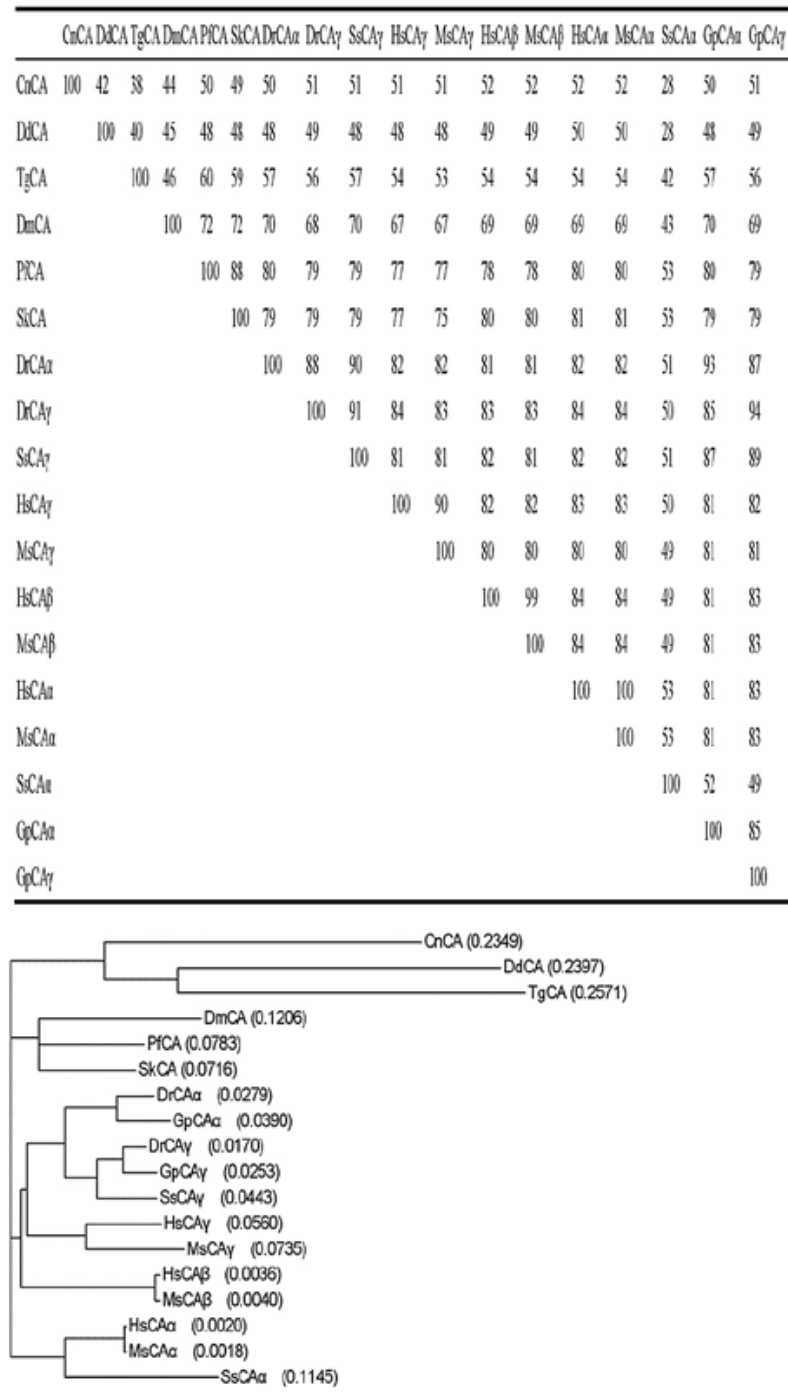

Figure 2. Phylogenetic tree analysis of G. przewalskii. Calcineurin A with those of other species. The phylogenetic tree was generated by using NJ method. GenBank accession numbers for these amino acid sequences of Calcineurin A are as follows: CnCA, CNA [Cryptococcus neoformans var. grubii] (AAB97372.1); DdCA, CNA [Dictyostelium discoideum AX4] (XP_642811.1); TgCA, CNA [Toxoplasma gondii] (AAM97278.1); DmCA, CNA [Drosophila melanogaster] (CAA54807.1); PfCA, CNA [Pinctada fucata] (EU797510); SkCA, CNA [Saccoglossus kowalevskii] (NP_001158479.1); $\operatorname{DrCA} \alpha$, CNA [Danio rerio, alpha isoform] (CAM46977.1); DrCA $\gamma$, CNA [Danio rerio, gamma isoform] (NP 001074063.2); SsCA $\gamma$, CNA [Salmo sala, gamma isoform] (ACI33493.1); HsCA $\gamma$, CNA [Homo sapiens, gamma isoform] (EAW63677.1); MsCA $\gamma$, CNA [Mus musculus, gamma isoform] (P48455.1); HsCA $\beta$, CNA [Homo sapiens, beta isoform] (EAW54497.1); MsCA $\beta$, CNA [Mus musculus, beta isoform] (P48453.2); $\mathrm{HsCA} \alpha$, CNA [Homo sapiens, alpha isoform] (EAX06124.1); $\mathrm{MsCA} \alpha, \mathrm{CNA}$ [Mus musculus, alpha isoform] (NP_032939.1); SsCA $\alpha$, CNA [Salmo sala, alpha isoform] (ACI67288.1); GpCA $\alpha$, CNA [Gymnocypris przewalskii, alpha isoform] (JQ407043); GpCA $\gamma$, CNA [Gymnocypris przewalskii, gamma isoform] (JQ407043)

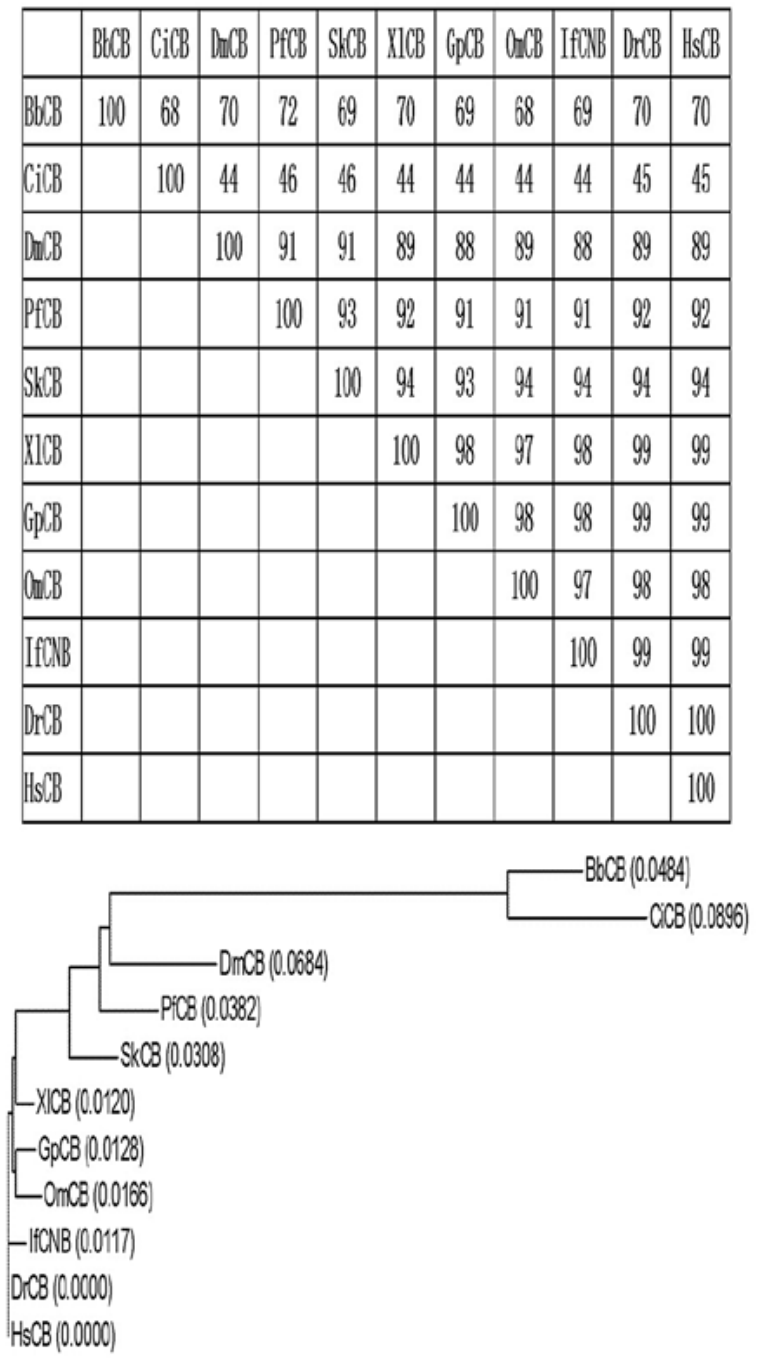

Figure 3. Phylogenetic tree analysis of G. przewalskii Calcineurin B with those of other species. GenBank accession numbers for these amino acid sequences of Calcineurin $\mathrm{B}$ are as follows: $\mathrm{BbCB}, \mathrm{CNB}$ [Beauveria bassiana] (ACU46638.1); CiCB, CNB [Coccidioides immitis RS] (XP 001248933.1); DrCB, CNB [Danio rerio] (NP 001004553.1); SsCB,CN B [Salmo salar] (NP_001134594.1); HsCB $\alpha, \mathrm{CNB}$ [Homo sapiens, alpha isoform] (EAW58961.1); $\mathrm{MmCB} \alpha, \mathrm{CNB}$ [Mus musculus] (EDL02306.1); DmCB, CNB [Drosophila melanogaster] (NP_524741.1); PfCB, CNB [Pinctada fucata] (ACI96107.1); SkCB, CNB [Saccoglossus kowalevskii] (NP 001161511.1); HsCB $\beta, \mathrm{CNB}$ [Homo sapiens, beta isoform] (EAW99878.1); GpCB, CNB [Gymnocypris przewalskii] (JQ410473); OmCB, CNB [Oncorhynchus mykiss] (NP_001154040.1)

$G p C A \gamma$ and $G p C B$ share the same trend after fertilization. From multicellular stage to organogenisis, $G p C A \alpha$ was expressed constitutively and declined from blastula stage, and reached its lowest level at neurula stage. In contrast, the expression level of $G p$ - 

ATGGGAAATGAGGCGAGTTATCCCTTGGAGATGTGCACACACTTTGACGCTGATGAGATTAAAAGACTAGGGAAGCGGTTTAAGAAACTC $\begin{array}{lllllllllllllllllllllllllllllll}\text { II } & G & \text { N } & \text { E } & \text { A } & \text { S } & \text { Y } & \text { P } & \text { L } & \text { E } & \text { II } & \text { C } & \text { T } & \text { H } & \text { F } & \text { D } & \text { A } & \text { D } & \text { E } & \text { I } & \text { K } & \text { R } & \text { L } & \text { G } & \text { K } & \text { R } & \text { F } & \text { K } & \text { K } & \text { L }\end{array}$ GACCTGGATAACTCGGGTTCTCTCAGCGTGGAGGAGTTTATGTCTCTACCCGAGTTGCAGCAGAATCCGCTGGTGCAGCGAGTCATCGAT

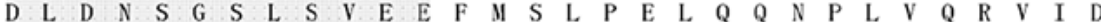
ATACTCGACACAGACGGAAATGGAGAAGTGGACTTTAAAGAGTTCATTGAAGGCGTCTCTCAGTTCAGTGTCAAGGGCGACAAGGAGCAG $\begin{array}{llllllllllllllllllllllllllllll}\text { I } & L & D & \text { T } & \text { D } & G & \text { N } & G & \text { E } & \text { V } & \text { D } & \text { F } & \text { K } & \text { E } & \text { F } & \text { I } & \text { E } & G & V & \text { S } & Q & \text { F } & \text { S } & \text { V } & \text { K } & \text { G } & \text { D } & \text { K } & \text { E } & Q\end{array}$ AAGCTCCGCTTTGCGTTCAGGATTTATGACATGGATAAGGATGGCTACATATCCAACGGTGAGCTGTTCCAGGTGCTGAAGATGATGGTA

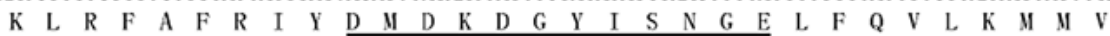
GGGAATAACCTGAAGGACACCCAGCTGCAGCAGATCGTCGACAAAACCATCATCAACGCAGACAAGGACGGGGACGGGAGGATATCTTTC $\begin{array}{llllllllllllllllllllllllllllll}G & N & N & \text { L } & K & \text { D } & \text { T } & Q & \text { L } & Q & Q & \text { I } & \text { V } & \text { D } & \text { K } & \text { T } & \text { I } & \text { I } & \text { N } & \text { A } & \text { D } & \text { K } & \text { D } & \text { G } & \text { D } & \text { G } & \text { R } & \text { I } & \text { S } & \text { F }\end{array}$ GAGGAGTTCTGCCTTGTGGTTGGTGGCTTAGACATTCACAAAAAGATGGTGGTGGACGTGTGA

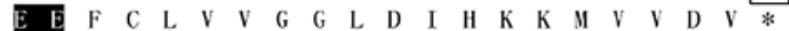

ATGGGAAATGAGGCGAGTTATCCCTTGGAGATGTGCACACACTTTGACGCTGATGAGATTAAAAGACTAGGGAAGCGGTTTAAGAAACTC GACCTGGATAACTCGGGTTCTCTCAGCGTGGAGGAGTTTATGTCTCTACCCGAGTTGCAGCAGAATCCGCTGGTGCAGCGAGTCATCGAT ATACTCGACACAGACGGAAATGGAGAAGTGGACTTTAAAGAGTTCATTGAAGGCGTCTCTCAGTTCAGTGTCAAGGGCGACAAGGAGCAG AAGCTCCGCTTTGCGTTCAGGATTTATGACATGGATAAGGATGGCTACATATCCAACGGTGAGCTGTTCCAGGTGCTGAAGATGATGGTA GGGAATAACCTGAAGGACACCCAGCTGCAGCAGATCGTCGACAAAACCATCATCAAOGCAGACAAGGACGGGGACGGGAGGATATCTTTC GAGGAGTTCTGCCTTGTGGTTGGTGGCTTAGACATTCACAAAAAGATGGTGGTGGACGTGTGA

Figure 4. cDNA sequences and deduced amino acid sequences of $G p C B$ : The four EF-hand type calcium-binding sites from 1 to 4 in $G p C B$ are lightly shaded, underlined, bold underlined and black shaded, respectively

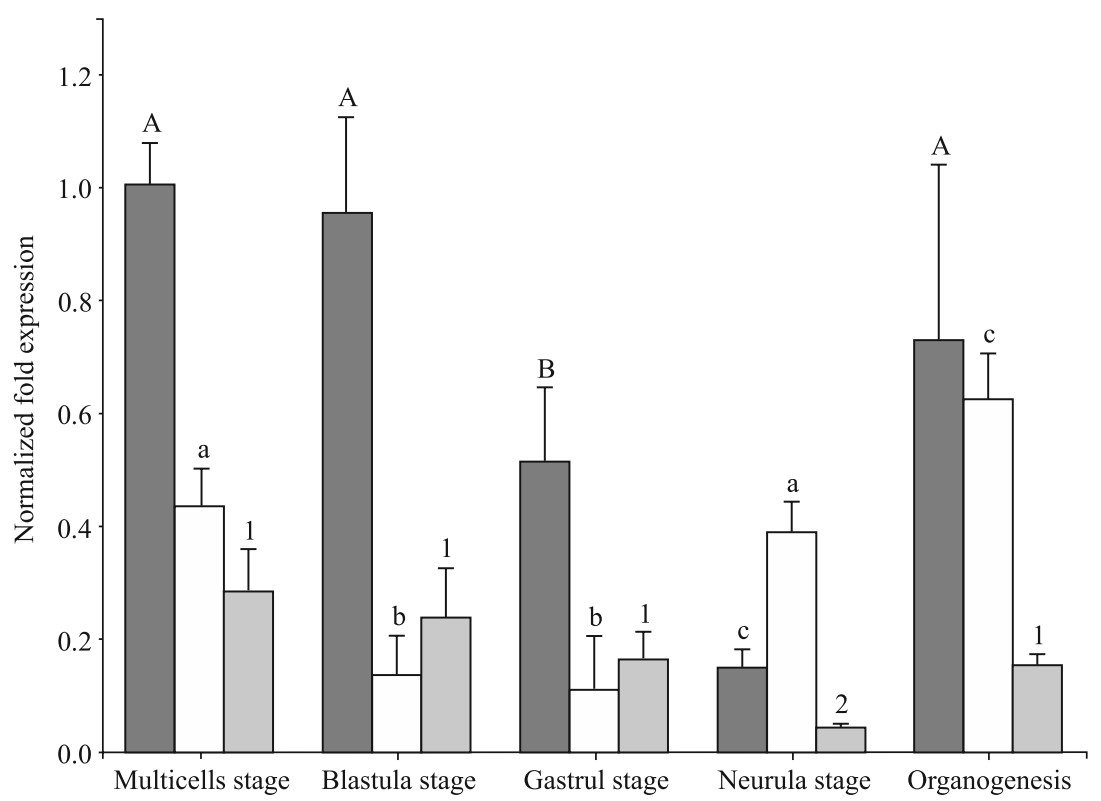

Figure 5. Real-time RT-PCR assays of $G$. przewalskii Calcineurin $m R N A$ in discrete stage of embryogenesis. Relative mRNA expressions were determined by the comparative $\mathrm{Ct}$ method considering PCR efficiency based on the normalization against the expression of $\beta$-actin in each sample. Fold increases (Mean \pm SV based on triplicate assays) were represented in histograms (dense for $G p C A \alpha$, none for $G p C A \beta$ and sparse for $G p C B$ ). Identical letters on the histograms indicate that the means were not statistically different based on ANOVA followed by Duncan's multiple range tests at $p=0.05$

$C A \gamma$ reached the lowest level at blastula and gastrula stage, and $G p C B$ showed minimum expression level at the neural stage.

In all animals, the earliest stages of embryonic development rely on maternal gene products, which are present in the egg at the time of fertilization. With the development of embryo in the later stage, transcription of the zygotic genome is gradually activated. It means that the maternal gene products and zygote transcriptional products coordinately regulate the embryonic development. According to our results, $\mathrm{CN}$ three subunits are expressed constitutively during various developmental stages. All of them showed higher expression level at the beginning stage. In the later stage of development, their expression level declined and reached to the minimum expression level. Finally, at the organogenisis stage the three studied genes' expression recovered quickly to the same level to that at the beginning stage of embryonic development. The expression pattern of $\mathrm{CN}$ three 
subunits demonstrated that $\mathrm{CN}$ is essentially needed in the whole process of embryonic development in naked carp, although their expression level showed a transient decrease probably due to the transitional period of maternal control of embryonic development and the onset of zygotic transcription.

\section{Immunohistochemical characterization of GpCA presence in naked carp's tissues}

Calcineurin is widely distributed in mammalian tissues, including brain, bone osteoclasts, heart, kidney, liver, and others. In naked carp, we detected that $\mathrm{CN}$ localized in separate tissues including kidney, brain, heart, gill, and spermary.

In the kidney of naked carp, $\mathrm{CN}$ was specifically detected in the epithelial cells in renal tubule (Figure 6A) which are adjoining renal capsules and play important roles in ion reabsorption and waste products secretion. It was found that $\mathrm{CN}$ inhibitors can increase salt-sensitivity of the kidney after transplantation [34]. Distinctive distribution of $\mathrm{CN}$ demonstrates that it participates in the renal physiology.

In the heart of naked carp, immunopositive signals congregated in the middle of the organ and formed several branching regions as showed in Figure 6B. After dephosphorylated by $\mathrm{CN}$, the member of NFAT family is translocated from the cytosol to the nucleus of cells where NFAT promotes gene transcription. Those transcribed genes play an important part in the development of cardiac hypertrophy [35-38]. In addition, cardiomyocytes can control their local neural milieu by expression of nerve growth factor (NGF) through calcineurin-NFAT pathway, which triggers sympathetic neural growth [39]. These results implied functions of $\mathrm{CN}$ specified heart areas also in naked carp.

In spermary of naked carp, the staining intensity distributed around the edge of seminiferous lobules occupied by spermatocytes (Figure 6C). In mammalian, testis-specific isoform of $\mathrm{CN}$ is expressed when meiosis begins, and increases in amount depending on the maturation of spermatogenesis [40]. In situ hybridization has shown this specific $\mathrm{CN}$ isoform was specifically localized to spermatocytes where meiosis occurs, but none or very few have been observed in spermatogonia, spermatids [41]. Imunohistochemical localization of $\mathrm{CN}$ in the mouse testis further confirms that it simply accumulates in spermatids nuclei and abundant increases during the initial stage of nuclear elongation, with almost no signal present in the cytoplasm [42]. All of the results suggest that $\mathrm{CN}$ in the testis is related to spermatogenesis.
In the brain of naked carp, positive signals appeared at the edge of the cerebral cortex as showed in Figure 6D. Calcineurin immunoreactivity was present in neurons, but a marked regional variation in strength of the immunoreactivity was observed; the caudatoputamen, hippocampal formation, and substantia nigra were intensely stained [43, 44]. Many studies have shown that $\mathrm{CN}$ takes part in the regulation of $\mathrm{Ca}^{2+}$ channel activity [45, 46], signal transduction at synapses and age-related changes in cognition during the aging process $[47,48]$, alteration of synaptic function and memory $[49,50]$. Together, $\mathrm{CN}$ may be a useful target for treatment of age-related memory impairments and neurodegenerative disease.

In the case of gill, strong signals distributed in the epithelial cells of the branchial filaments and the base of gill filaments as showed in Figure 6E. Gills perform a variety of physiological functions in adult fish including respiratory gas exchange, ion and water balance, excretion of nitrogenous wastes and the maintenance of acid-base balance. In the gill, $\mathrm{Ca}^{2+}$-ATPases modulate the inward flux of $\mathrm{Ca}^{2+}$ into gill epithelial cells $[51,52]$, and may play a part in gill acclimation responses to environmental $\mathrm{Ca}^{2+}$ changes and osmoregulation [53]. The presence of $\mathrm{CN}$ in naked carp's gill suggests that also in this teleost fish $\mathrm{CN}$ may play a key role in calcium uptake and accumulation.

As mentioned above, $\mathrm{CN}$ is localized in various cells of different tissues. The function of $\mathrm{CN}$ especially in the immune system, osteoclast bone resorption, cardiac hypertrophy, skeletal muscle hypertrophy, spermatid motility, apoptosis, hormone secretion and other processes has been described in many studies. In light of the demonstrated in this study immunohistological localization of $G p \mathrm{CA}$, it is implicated that the function of $\mathrm{CN}$ in naked carp from Lake Qinghai needs to be further investigated.

\section{Conclusions}

In this study, we have isolated cDNA sequences of paralogue isoforms of $\mathrm{CN}$ catalytic subunits and $\mathrm{CN}$ regulatory subunit from Gymnocypris przewalskii. Gene expression profiles and immunohistochemistry analysis demonstrate that $\mathrm{CN}$ distribute ubiquitously in early embryonic stages and in various tissues. Our findings provide some important clues for further understanding the physiological characteristics of naked carp during embryonic development and in adaptation to the environment changes.

\section{Acknowledgments}

This work was financially supported by the Program of National Fisheries Resources Platform of China, the 

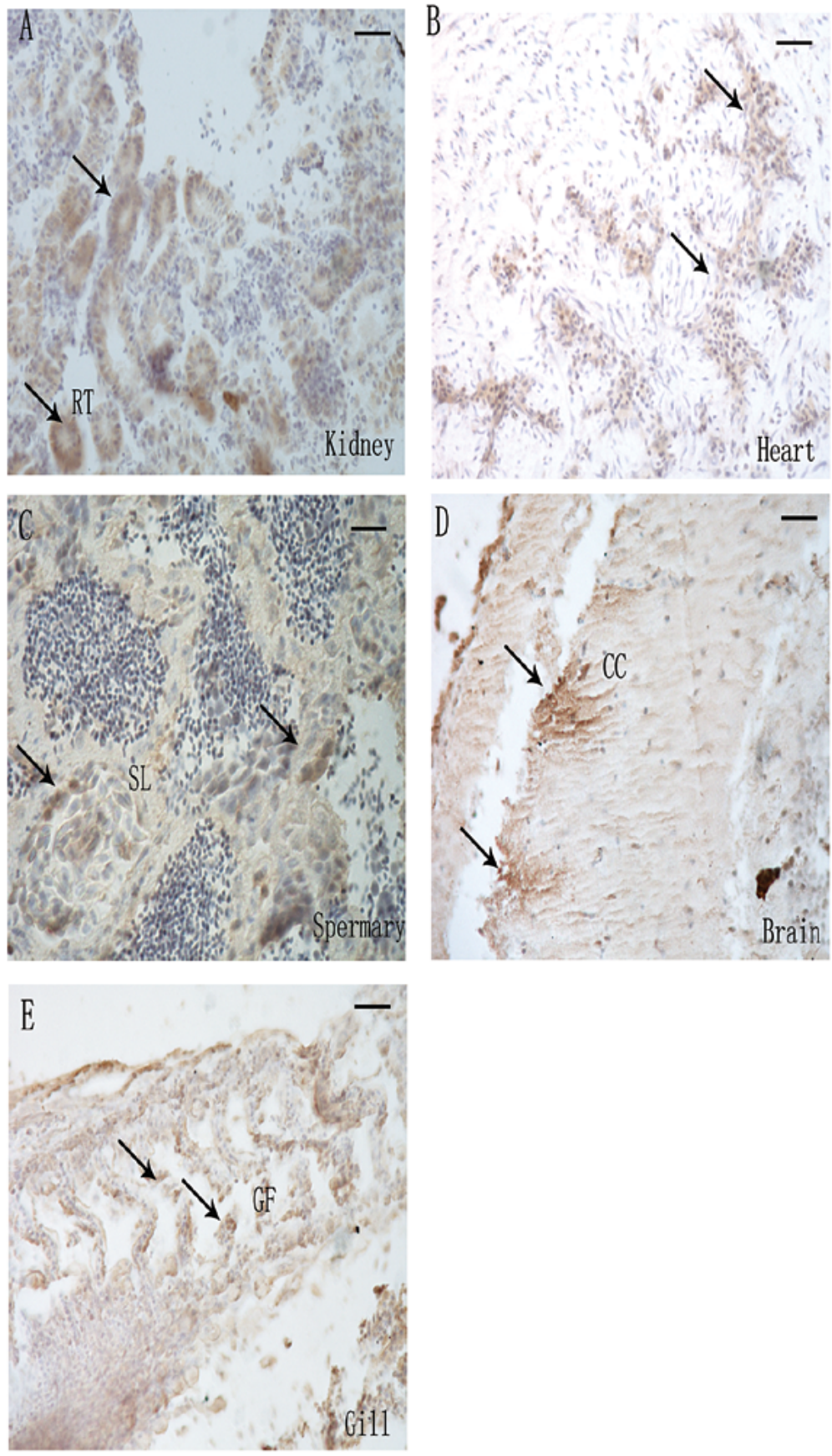

Figure 6. Immunohistochemical localization of CNA in diverse tissues of G. przewalskii. A. Kidney; B. Heart; C. Spermary; D. Brain; E. Gill. Immunostaining signals are indicated by arrow. Bars $100 \mu \mathrm{m}$ in (B), (E); $50 \mu \mathrm{m}$ in (A), (C) and (D). RT — renal tubule; SL — seminiferous lobule; CC — cerebral cortex; GF — gill filament

applied background research project from Qinghai Science and Technology Department, Qinghai province, China (2013-Z-715), and the research project at Qinghai University (2011-QNZ-1).

\section{References}

1. Yang J, Qi H, Shi J, Chen D, Huang F, Tang H. Hydrochemistry properties and water quality analysis of Qinghai hu lake. Freshwtr Fish. 2005;35:28-32.
2. Shi J, Y J, Qi H, Chen D, Liu S, Duan X. Evaluation and analysis of Gymnocypris przewalskii resources. Freshwtr Fish. 2000;30:38-40.

3. Chen D, Z X, Tan X, Wang K, Qiao Y, Chang Y. Hydroacoustic study of spatial and temporal distribution of Gymnocypris przewalskii (Kessler, 1876) in Qinghai Lake, China. Environ Biol Fishes. 2009;84:231-239.

4. Walker K, Dunn I, Edwards D, Petr T, Yang H. A fishery in a changing lake environment: The naked carp (Gymnocypris przewalskii); (Kessler) (Cyprinidae: schizothora- 
cinae) in Qinghai Hu, China. Int J Salt Lake Res. 1995;4: 169-222.

5. Walker K, Yang H. Fish and fisheries of western China. Fish and Fisheries at Higher Altitudes: Asia. 1999;385:304.

6. Wang Y, Gonzalez R, Patrick M et al. Unusual physiology of scale-less carp, Gymnocypris przewalskii, in Lake Qinghai: a high altitude alkaline saline lake. Comp Biochem Physiol A Mol Integr Physiol. 2003;134:409-421.

7. Wood C, Du J, Rogers J et al. Przewalski's naked carp (Gymnocypris przewalskii): an endangered species taking a metabolic holiday in Lake Qinghai, China. Physiol Biochem Zool. 2007;80:59-77.

8. Klee C, Haiech J. Concerted role of calmodulin and calcineurin in calcium regulation. Ann New York Acad Sci. 1980;356:43-54.

9. Klee C, Ren H, Wang X. Regulation of the Calmodulin-stimulated Protein Phosphatase, Calcineurin. J Biol Chem. 1998;273:13 367-13 370.

10. Stemmer P, Klee C. Dual calcium ion regulation of calcineurin by calmodulin and calcineurin B. Biochemistry. 1994;33:6859-6866.

11. Steinhardt R, Epel D. Activation of sea-urchin eggs by a calcium ionophore. Proc Natl Acad Sci U.S.A. 1974;71:1915-1919.

12. Uryu M, Nakatomi A, Watanabe M, Hatsuse R, Yazawa M. Molecular cloning of cDNA encoding two subunits of calcineurin from scallop testis: demonstration of stage-specific expression during maturation of the testis. J Biochem. 2000;127:739-746.

13. Boutet I, Moraga D, Marinovic L, Obreque J, Chavez-Crooker P. Characterization of reproduction-specific genes in a marine bivalve mollusc: influence of maturation stage and sex on mRNA expression. Gene. 2008;407:130-138.

14. Li X, Zhu L, Yang A et al. Calcineurin-NFAT signaling critically regulates early lineage specification in mouse embryonic stem cells and embryos. Cell Stem Cell. 2011;8:46-58.

15. Muller M, Sasaki Y, Stevanovic I et al. Requirement for balanced $\mathrm{Ca} / \mathrm{NFAT}$ signaling in hematopoietic and embryonic development. Proc Natl Acad Sci U.S.A. 2009;106:7034-7039.

16. Kinikoglu B, Kong Y, Liao E. Characterization of cultured multipotent zebrafish neural crest cells. Exp Biol Med. 2014;239:159-168.

17. Li C, Huang J, Li S et al. Cloning, characterization and immunolocalization of two subunits of calcineurin from pearl oyster (Pinctada fucata). Comp Biochem Physiol B Biochem Mol Biol. 2009;153:43-53.

18. Li C, Hu Y, Liang J et al. Calcineurin plays an important role in the shell formation of pearl oyster (Pinctada fucata). Mar Biotechnol (NY). 2010;12:100-110.

19. Kong Y, Jing G, Yan $\mathrm{Z}$ et al. Cloning and characterization of Prisilkin-39, a novel matrix protein serving a dual role in the prismatic layer formation from the oyster Pinctada fucata. J Biol Chem. 2009;284:10 841-10 854.

20. Gasteiger E, Hoogland C, Gattiker A et al. Protein identification and analysis tools on the ExPASy server. In: J.M. Walker ed. The Proteomics Protocols Handbook. Louisville, KY: Humana Press; 2005;571-607.

21. Marchler-Bauer A, Anderson J, Cherukuri P et al. CDD: a Conserved Domain Database for protein classification. Nucleic Acids Res. 2005;33:D192-D196.

22. de Castro E, Sigrist C, Gattiker A et al. ScanProsite: detection of PROSITE signature matches and ProRule-associated functional and structural residues in proteins. Nucleic Acids Res. 2006;34:W362-W365.
23. Muramatsu T, Kincaid R. Molecular cloning and chromosomal mapping of the human gene for the testis-specific catalytic subunit of calmodulin-dependent protein phosphatase (calcineurin A). Biochem Biophys Res Commun. 1992;188:265-271.

24. Buttini M, Limonta S, Luyten M, Boddeke H. Distribution of calcineurin A isoenzyme mRNAs in rat thymus and kidney. Histochem. 1995;27:291-299.

25. Kuno T, Mukai H, Ito A et al. Distinct cellular expression of calcineurin A alpha and A beta in rat brain. J Neurochem. 1992;58:1643-1651.

26. Gooch J. An emerging role for calcineurin A alpha in the development and function of the kidney. Am J Physiol Renal Physiol. 2006;290:F769-F776.

27. Gooch J, Toro J, Guler R, Barnes J. Calcineurin A-alpha but not A-beta is required for normal kidney development and function. Am J Pathol. 2004;165:1755-1765.

28. Yap K, Ames J, Swindells M, Ikura M. Diversity of conformational states and changes within the EF-hand protein superfamily. Proteins: Structure, Function, and Bioinformatics. 1999;37:499-507.

29. Ueki K, Kincaid R. Interchangeable associations of calcineurin regulatory subunit isoforms with mammalian and fungal catalytic subunits. J Biol Chem. 1993;268:6554-6559.

30. Steinhardt R, Zucker R, Schatten G. Intracellular calcium release at fertilization in the sea urchin egg. Devel Biol. 1977;58:185-196.

31. Mei W, Lee K, Marlow F, Miller A, Mullins M. hnRNP I is required to generate the $\mathrm{Ca}^{2+}$ signal that causes egg activation in zebrafish. Development. 2009;136:3007-3017.

32. Nishiyama T, Yoshizaki N, Kishimoto T, Ohsumi K. Transient activation of calcineurin is essential to initiate embryonic development in Xenopus laevis. Nature. 2007;449:341-345.

33. Yoshida Y, Kim S, Chiba K, Kawai S, Tachikawa H, Takahashi N. Calcineurin inhibitors block dorsal-side signaling that affect late-stage development of the heart, kidney, liver, gut and somitic tissue during Xenopus embryogenesis. Dev Growth Differ. 2004;46:139-152.

34. Hoorn E, Walsh S, Unwin R, Ellison D. Hypertension after kidney transplantation: Calcineurin inhibitors increase salt-sensitivity. J Hypertens. 2012;30:832-833.

35. Molkentin J. Calcineurin-NFAT signaling regulates the cardiac hypertrophic response in coordination with the MAPKs. Cardiovasc Res. 2004;63:467-475.

36. Molkentin J, Lu J, Antos C et al. A calcineurin-dependent transcriptional pathway for cardiac hypertrophy. Cell. 1998;93:215-228.

37. Wilkins B, Dai Y, Bueno O et al. Calcineurin/NFAT coupling participates in pathological, but not physiological, cardiac hypertrophy. Circ Res. 2004;94:110-118.

38. Wilkins B, De Windt L, Bueno O et al. Targeted disruption of NFATc3, but not NFATc4, reveals an intrinsic defect in calcineurin-mediated cardiac hypertrophic growth. Mol Cell Biol. 2002;22:7603-7613.

39. Rana O, Saygili E, Meyer C et al. Regulation of nerve growth factor in the heart: the role of the Calcineurin-NFAT pathway. J Mol Cell Cardiol. 2009;46:568-578.

40. Nishio H, Matsui H, Moia L et al. The evidence for post -meiotic expression of a testis-specific isoform of a regulatory subunit of calcineurin using a monoclonal antibody. Biochem Biophys Res Commun. 1992;187:828-831.

41. Miyamoto K, Matsui H, Tomizawa K et al. In situ localization of rat testis-specific calcineurin $\mathrm{B}$ subunit isoform beta 1 in 
the developing rat testis. Biochem Biophys Res Commun 1994;203:1275-1283.

42. Moriya M, Fujinaga K, Yazawa M, Katagiri C. Immunohistochemical localization of the calcium/calmodulin-dependent protein phosphatase, calcineurin, in the mouse testis: its unique accumulation in spermatid nuclei. Cell Tissue Res. 1995;281:273-281.

43. Goto S, Matsukado Y, Mihara Y, Inoue N, Miyamoto E. Calcineurin in human brain and its relation to extrapyramidal system. Immunohistochemical study on postmortem human brains. Acta Neuropathol. 1986;72:150-156.

44. Goto S, Matsukado Y, Mihara Y, Inoue N, Miyamoto E. The distribution of Calcineurin in rat brain by light and electron microscopic immunohistochemistry and enzyme-immunoassay. Brain Res. 1986;397:161-172.

45. Armstrong D. Calcium channel regulation by calcineurin, a $\mathrm{Ca}^{2+}$-activated phosphatase in mammalian brain. Trends Neurosci. 1989;12:117-122.

46. Loane D, Hicks GA, Perrino B, Marrion N. Inhibition of BK channel activity by association with calcineurin in rat brain. Eur J Neurosci. 2006;24:433-441.

47. Agbas A, Zaidi A, Michaelis E. Decreased activity and increased aggregation of brain calcineurin during aging. Brain Res. 2005;1059:59-71.
48. Foster T, Sharrow K, Masse J, Norris C, Kumar A. Calcineurin links $\mathrm{Ca}^{2+}$ dysregulation with brain aging. J Neurosci. 2001;21:4066-4073.

49. Mansuy I, Mayford M, Jacob B, Kandel E, Bach ME. Restricted and regulated overexpression reveals calcineurin as a key component in the transition from short-term to long-term memory. Cell. 1998;92:39-49.

50. Winder D, Mansuy I, Osman M, Moallem T, Kandel E. Genetic and pharmacological evidence for a novel, intermediate phase of long-term potentiation suppressed by calcineurin. Cell. 1998;92:25-37.

51. Shahsavarani A, Perry S. Hormonal and environmental regulation of epithelial calcium channel in gill of rainbow trout (Oncorhynchus mykiss). Am J Physiol Regul Integr Comp Physiol. 2006;291:R1490-R1498.

52. Viarengo A, Mancinelli G, Pertica M, Fabbri R, Orunesu M. Effects of heavy metals on the $\mathrm{Ca}^{(2+)}$-ATPase activity present in gill cell plasma-membrane of mussels (Mytilus galloprovincialis Lam.). Comp Biochem Physiol C. 1993;106:655-660.

53. Pinto $\mathrm{P}$, Matsumura $\mathrm{H}$, Thorne $\mathrm{M}$ et al. Gill transcriptome response to changes in environmental calcium in the green spotted puffer fish. BMC Genomics. 2010;11:476.

Submitted: 8 April, 2014

Accepted after reviews: 16 September, 2014 